\title{
Pathomorphological and Molecular Detection of Canine Monocytic Ehrlichiosis in a Siberian Husky
}

\author{
N. Jayanthi ${ }^{1 *}$, R. Saahithya ${ }^{1}$, L.J. Harish ${ }^{2}$ and P. Azhahianambi ${ }^{3}$ \\ ${ }^{1}$ Department of Veterinary Pathology, ${ }^{2}$ Department of Veterinary Clinical Medicine, \\ Madras Veterinary College, Chennai - 600 007, India \\ ${ }^{3}$ Department of Veterinary Parasitology, Madras Veterinary College, Chennai - 600007 \\ *Corresponding author
}

\section{A B S T R A C T}

\begin{abstract}
Keywords
Ehrlichia canis,

Dog,

Pathomorphology,

Nested PCR,

Histopathology

Article Info

Accepted:

06 June 2018

Available Online:

10 July 2018

A three-year-old Siberian husky was brought for necropsy with a history of respiratory distress and erythematous patches on the ventral abdomen. Postmortem examination revealed icteric mucous membranes, diffuse erythematous patches in the ventral abdomen and ticks all over the body. The intermediate and diaphragmatic lobe of the lungs revealed haemorrhages. Liver was mottled and tan yellow brown. Gall bladder had a solitary calculus. Spleen was mildly swollen, dark brown and congested. Kidneys had a pitted and granular cortical surface with hemorrhages in the pelvis. Severe congestion of the cerebral vessels was observed. The right inner thigh muscles revealed severe paint brush hemorrhages and extended deep into the gluteal muscles. Histopathological examination revealed congested liver, necrotic changes in the kidney, focal interstitial nephritis and eosinophilic exudate in distal convoluted tubule, myocardial degeneration, congested spleen with hemosiderin pigments and mild neuronal degeneration. Nested PCR was carried out on the liver samples and was positive for Ehrlichia canis.
\end{abstract}

\section{Introduction}

Canine monocytic Ehrlichiosis (CME) is a globally distributed vector borne disease transmitted by ticks. The main tick vector involved in the transmission is Rhiphicephalus sanguineous which is often called brown dog tick. It is an obligate intracellular gram negative bacteria belonging to the family Anaplas mataceae. It causes multisystemic disease in the affected host (Barman et al.,
2014; Waner and Harrus, 2013). The disease gets manifested in a variety of clinical signs. The major phases are acute, subclinical and chronic. Ehrlichia tends to cause persistent infection in their natural host. Most of the affected dogs recover after the acute infection, however they reach a subclinical state. But, in certain cases the chronic state of the infection is evident in the form of thrombocytopenia, pancytopenia and hyperglobulinemia. In most of the cases, dogs may die due to severe 
bleeding tendencies or sepsis or both (Mylonakis et al., 2004). The clinicopathological signs may vary based on the strain of the Ehrlichia sp. and its respective geographic distribution (Lakkawar et al., 2003).

Clinically, the organism can be detected by the presence of morulae in the cytoplasm of the monocytes. However, the success rate of detection of the parasite through blood smear is unrewarding in most of the cases (Cohn, 2003). In the recent years, advanced serological tests like polymerase chain reaction, Immunofluroscent antibody technique (IFAT), DOT ELISA are highly prevalent. The stage of the clinical infection should be noted while involving the diagnostic tests in order to elucidate the organism without obtaining false positive results (Wen et al., 1997; Frank and Breitschwerdt, 1999).

The present report describes the clinicopathological, molecular detection and pathomorphology of monocytic ehrlichiosis in a siberian husky.

\section{Materials and Methods}

\section{Necropsy examination}

A three year old female Siberian husky was admitted for necropsy examination to the Department of Veterinary Pathology, Madras Veterinary College, Chennai - 600007 with a history of sudden death. Preliminary history was obtained and necropsy examination was carried out.

\section{Collection of samples}

The tissue samples of $4 \mathrm{~mm}$ thickness of each were collected from liver, heart, kidney, brain, lymphnodes and pancreas in 10 per cent formalin in wide mouthed bottles. After fixation, the samples were processed for histopathology. Paraffin embedded tissue sections were cut into $4 \mu \mathrm{m}$ thickness and were stained with haematoxylin and eosin (H\&E). The slides were viewed under light microscopy. The multiple pieces of liver were collected in freeze packs for polymerase chain reaction and stored in $-20^{\circ} \mathrm{C}$ until further analysis.

Heart blood smears and impression smear from spleen were collected in a clean grease free microscopic slide. It was air dried and stained with Leishman Giemsa stain solution for 30 minutes, air dried and viewed under oil immersion objective of light microscope.

\section{Nested Polymerase Chain Reaction}

DNA isolation kit (QIAamp DNA Mini Kit®, Qiagen, Shriram Enterprises) was used for the parasite DNA extraction from liver tissue collected in freeze packs according to the manufacturer's instructions. Genomic DNA was also isolated from the whole blood of infection-free dog and used as a negative control along with nuclease-free water.

Nested PCR for the amplification of the 16s rRNA gene fragment of E.canis was employed following the procedure of Rajagopal et al. (2009) and Birkenheuer et al. (2003) respectively. Following primers were used:

\begin{tabular}{|c|c|c|c|}
\hline \multicolumn{4}{|c|}{ a. Ehrlichia spp. (16s rRNA gene) } \\
\hline Primer name & Sequence & & Size \\
\hline ECC & 5' AGA ACG GCT GGC GGC MG C 3'’ & \multirow{2}{*}{$1^{\text {st }}$ cycle } & \multirow{4}{*}{$387 \mathrm{bp}$} \\
\hline ECB & 5' CGT ATT ACC GCG GCT GCT GGC A 3' & & \\
\hline ECAN5 & 5' CAA TTA TTT ATA GCC TCT GGC TAT AGG A 3' & \multirow{2}{*}{$2^{\text {nd }}$ cycle } & \\
\hline HE3 & 5' TAT AGG TAC CGT CAT TAT CTT CCC TAT 3’ & & \\
\hline
\end{tabular}


Thermal cycling for PCR amplification was as follows:

$\frac{94^{\circ} \mathrm{C}}{3 \min } \rightarrow\left[\frac{94^{\circ} \mathrm{C}}{1 \min } \rightarrow \frac{65^{\circ} \mathrm{C}}{2 \min } \rightarrow \frac{72^{\circ} \mathrm{C}}{2 \min }\right] \times 30$ cycles $\rightarrow \frac{72^{\circ} \mathrm{C}}{5 \min } \rightarrow \frac{4^{\circ} \mathrm{C}}{\infty}$

\section{Results and Discussion}

\section{Blood smear examination}

The heart blood smear revealed the presence of basophilic round to oval morula in the cytoplasm of the monocytes. About 20 per cent of the high power fields revealed the presence of E. canis morula (Fig.12).

\section{Haematology and serum biochemistry findings}

Haemogram revealed hypochromasia, relative monocytosis (10\%), severe thrombocytopenia (0.58 lakhs/cmm), activated platelets while serology revealed elevated BUN (144.39 $\mathrm{mg} / \mathrm{dl})$ and creatinine values $(8.16 \mathrm{mg} / \mathrm{dl})$.

\section{Gross pathology}

Postmortem examination revealed icteric mucous membranes (Fig. 1), diffuse erythematous patches in the ventral abdomen (Fig. 2) and ticks all over the body. Serosanguineous fluid was found in the abdominal cavity $(150 \mathrm{~mL})$, blood in the pericardial sac (Fig. 10), flabby right ventricular myocardium. Trachea revealed frothy exudate at the bifurcation. Lungs were congested, edematous and hemorrhagic in the intermediate and diaphragmatic lobe which sank in water. Liver was congested, mottled, tan yellow brown in color (Fig. 4). Gall bladder had a solitary calculus (Fig. 5). Spleen was mildly swollen, dark brown and congested (Fig. 6). Kidneys had a pitted and granular cortical surface with hemorrhages in the pelvis (Fig. 8). Intestines revealed severe hemorrhages (Fig. 7). Patchy hemorrhages were noticed in the pancreas, urinary bladder and uterus. Severe congestion of the cerebral vessels with haemorrhages into the parenchymawere noticed (Fig. 9). The right inner thigh muscles revealed severe paint brush hemorrhages and extended deep into the gluteal muscles (Fig. 3).

\section{Histopathology}

Histopathological examination revealed congested liver, necrotic changes in the kidney, focal interstitial nephritis and eosinophilic exudate in distal convoluted tubule (Fig. 15). Peripancreatic and periductal fibrosis in the pancreas (Fig. 16), congestion, hemorrhage and oedema in the lungs, myocardial degeneration (Fig. 13), congested spleen with hemosiderin pigments and mild neuronal degeneration, focal congestion and mild oedema were also observed (Fig. 14).

\section{Nested PCR}

Nested PCR reaction for the amplification of Ehrlichiacanis yielded a product size of $387 \mathrm{bp}$ confirming E.canis infection (Fig. 17).

The clinical signs and the haematobiochemical findings in the present case correlated with the earlier reports of Barman et al. (2014); Mylonakiset al. (2004); Lakkawar et al. (2003). In addition, they have related to the fact that anaemia, thrombocytopenia and subsequent bleeding tendencies might indicate poor prognosis of affected dogs. In addition, breeds like Siberian huskies are not suited for hot tropical climates and their thick fur coat are more prone for tick infestation. In this case, sudden death may be attributed to severe thrombocytopenia and extensive haemorrhages which was concentrated highly in the thigh region. 
Fig.1 Dog - Eye - Icteric mucous membrane; Fig.2 Dog - Diffuse erythematous patches in the ventral abdomen; Fig.3 Dog - The right inner thigh muscles revealed severe paint brush haemorrhages and extended deep into the gluteal muscles; Fig.4 Dog - Liver - Congested, mottled, tan yellow brown in colour; Fig.5 Dog - Gall bladder had a solitary calculus; Fig.6 Dog - Spleen - mildly swollen, dark brown and congested; Fig.7 Dog - Intestines revealed severe haemorrhages; Fig.8 Dog- Kidneys - haemorrhages in the pelvis; Fig.9 Dog - Brain - Severe congestion of the cerebral vessels with haemorrhages into the parenchyma;

Fig.10 Dog - Heart - About $30 \mathrm{ml}$ of blood in the pericardial sac
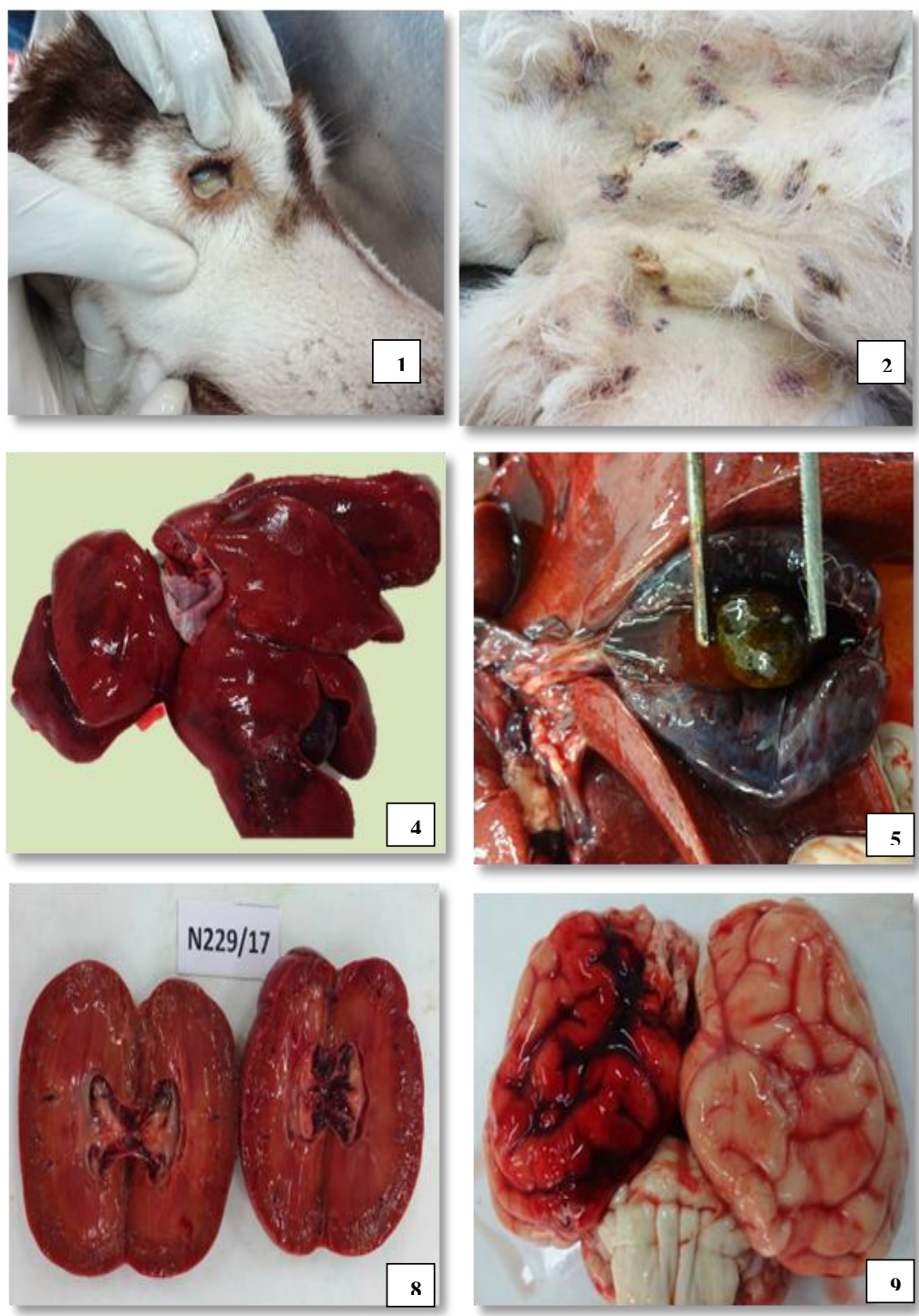
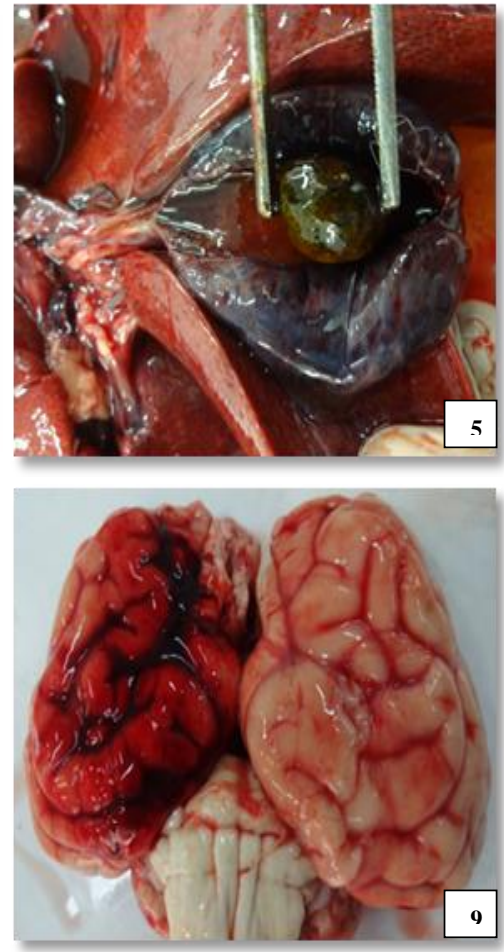
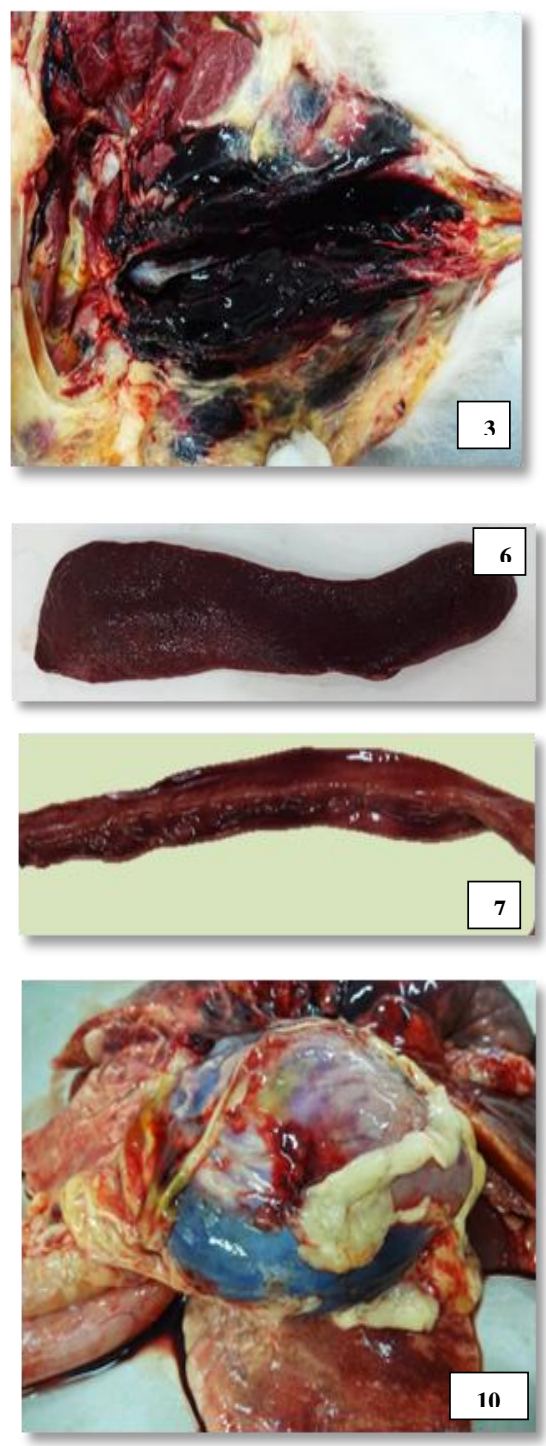
Fig.2 Dog - Heart blood smear - Presence of basophilic oval to round E. canis morula in the cytoplasm of the monocyte (Leishman \& Giemsa 100x); Fig.13 Heart - Myocardial degeneration (H\&E 20x); Fig.14 Brain - Mild neuronal degeneration (H\&E 20x); Fig.15 Kidneys - Necrotic changes with eosinophilic exudate in distal convoluted tubules (H\&E 20x); Fig.16 Pancreas Peripancreatic and periductal fibrosis (H\&E 20x); Fig.17 Nested PCR - Lane - 2: Brain, Lane3: Liver - Positive for Ehrlichiacanis at 387 bp.
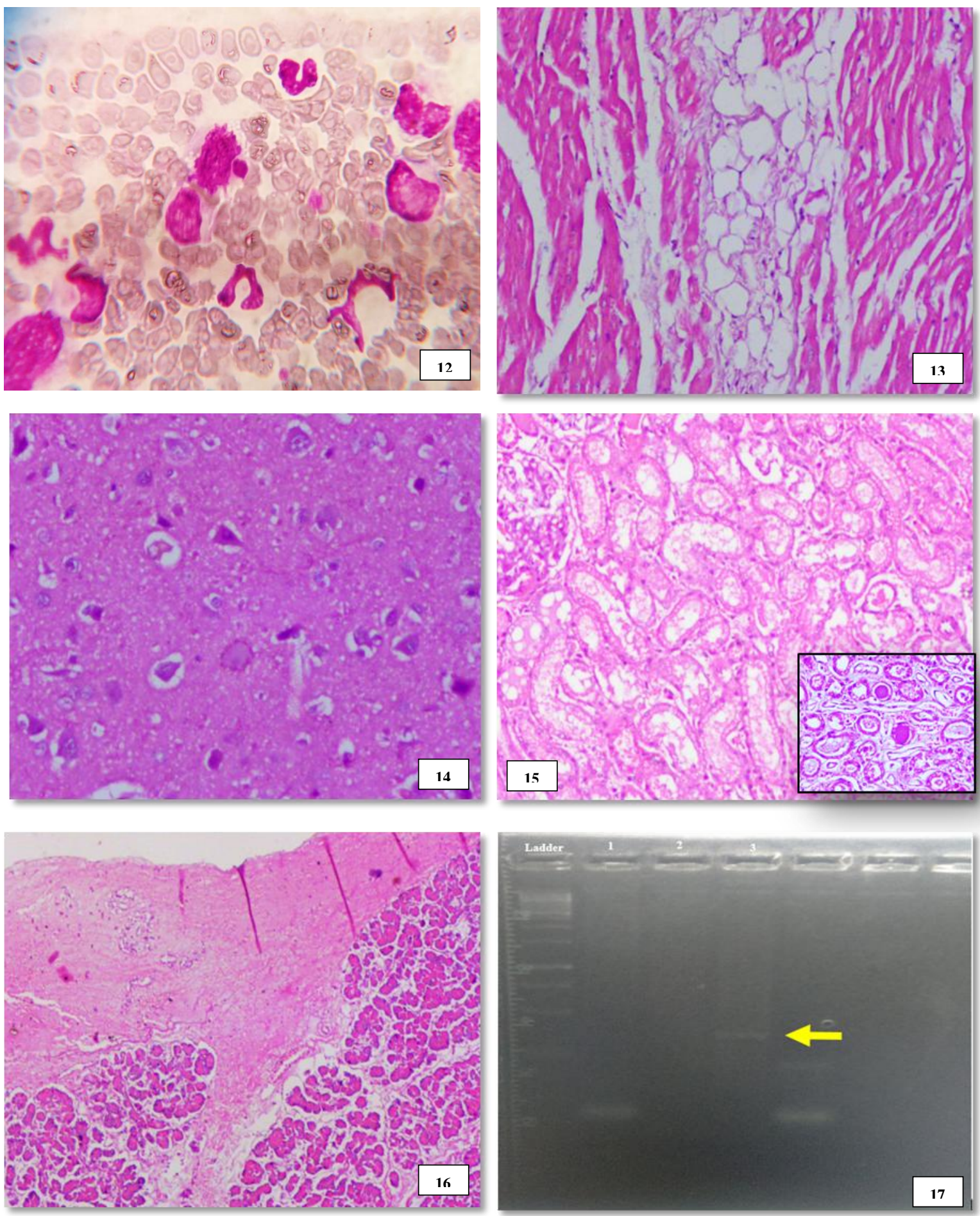
The evidence of morula, gross and histopathological changes was in correlation with the findings of Lakkawaret al.(2003); Waner and Harrus, (2013). They have reported that the variety of clinical and pathomorphological manifestations are due to the ubiquity of the organism. However, renal involvement in this case is in agreement with the findings of Silva et al.(2016) who reported an increased occurrence of glomerulonephritis in the spontaneously occurring cases of canine monocytic ehrlichiosis. This case represented involvement of interstitial nephritis with eosinophilic exudates in renal tubules and necrosis of tubular epithelium. The presence of cholelith in this case might be associated with jaundice. The incidence of cholelith associated with ehrlichiosis has not been reported.

The confirmation of the organism through nested PCR is in accordance with the results of Lakshmananet al.(2007); Wen et al.(1997); Olanoet al.(2004). They have reported that Nested PCR could sensitively detect the presence of the organism even in treated cases of ehrlichiosis and in cases where demonstration of the organism is not possible in blood smears. Hence, the present case concluded the successful detection of ehrlichiosis in a Siberian husky and its pathomorphological findings.

\section{Acknowledgements}

The authors gratefully acknowledge the support from the Department of Veterinary Parasitology and thank the facilities provided by the Tamil Nadu Veterinary and Animal Sciences University for carrying out the work.

\section{References}

Barman, D., Baishya, B.C., Sarma, D., Phukan, A., and Dutta, T.C.2014. A
Case Report of Canine Ehrlichia Infection in a Labrador Dog and its therapeutic Management. Bangladesh Journal of Veterinary Medicine. 12 (2): 237-239.

Cohn, L.A., 2003. Ehrlichiosis and related infections. Veterinary Clinics of North America Small Animal Practice. 33(4): 863-884.

Frank, J. R., and Breitschwerdt, E.B. 1999.A Retrospective Study of Ehrlichiosis in 62 Dogs from NorthCarolina and Virginia.Journal of Veterinary Internal Medicine.13:194-201.

Lakkawar, A.W., Nair, M.G., Varshney, K.C., Sreekrishnan, R., Rao, V.N. 2003.Pathologyof Canine Monocytic Ehrlichiosis Ina GermanShepherd Dog. Slovakian Veterinary Research. 40 (2): 119-128.

Lakshmanan, B., John, L., Gomathinayagam, S., and Dhinakarraj, G. 2007. Molecular detection of Ehrlichiacanis from blood of naturallyinfected dogs in India.Veterinarskiarhiv. 77 (4): 307312.

Mylonakis, M.E., Koutinas, A.F., Breitschwerdt, E.B., Hegarty, B.C., Billinis, C.D., Leontides, L.S., and Kontos, V.S. 2004.Chronic Canine Ehrlichiosis (Ehrlichia canis): A Retrospective Study of 19 Natural Cases. Journal of American Animal Hospital Association.40:174-184.

Olano, J.P., Wen, G., Feng, H.M., McBride, J. W., and Walker, D.H. 2004. Histologic, Serologic, and Molecular Analysis of Persistent Ehrlichiosis in a Murine Model.

American Journal of Pathology.165 (3): $997-$ 1006.

Silva, L. S., Pinho, F.A., Prianti, M.G., Braga, J.F.V., Pires, L.V., França, S.A., Silva, S.M.M.S. 2016. Renal histopathological changes in dogs naturally infected with Ehrlichia canis. 
Brazilian Journal of Veterinary Pathology. 9(1): $2-15$.

Waner, T., and Harrus, S. 2013.Canine Monocytic Ehrlichiosis - From Pathologyto Clinical Manifestations. Israel Journal of Veterinary Medicine.68 (1): 12-18.

Wen, B., Rikihisa, Y., Mott, J. M., Greene,
R., Kim, H.Y., Couto, G.C., Unver, A., and Bartsch, R. 1997. Comparison of Nested PCR with ImmunofluorescentAntibody Assay for Detection of Ehrlichia canis infection in Dogs Treated with Doxycycline. Journal of Clinical Microbiology.35 (7): 18521855.

\section{How to cite this article:}

Jayanthi, N., R. Saahithya, L.J. Harish and Azhahianambi, P. 2018. Pathomorphological and Molecular Detection of Canine Monocytic Ehrlichiosis in a Siberian Husky. Int.J.Curr.Microbiol.App.Sci. 7(07): 678-684. doi: https://doi.org/10.20546/ijcmas.2018.707.082 